\title{
Femoral Neuropathy Secondary to Autosomal Dominant Polycystic Kidney Disease: A Case Report
}

\author{
Jeehyun Yoo, MD, Kil-Byung Lim, MD, PhD, Hong-Jae Lee, MD, \\ Jiyong Kim, MD, Eun-Cheol You, MD, Joongmo Kang, MD
}

Department of Physical Medicine and Rehabilitation, Inje University Ilsan Paik Hospital, Goyang, Korea

\begin{abstract}
Compressive femoral neuropathy is a disabling condition accompanied by difficulty in hip flexion and knee extension. It may result from retroperitoneal hematoma or bleeding, or from complications associated with pelvic, hip surgery, and renal transplants. A 55-year-old female with autosomal dominant polycystic kidney disease presented with proximal muscle weakness in lower extremities. The patient experienced recurrent renal cyst infection, with aggravated weakness during each event. Electromyography and nerve conduction study revealed bilateral femoral neuropathy. Computed tomography and magnetic resonance images were added to further identify the cause. As a result, a diagnosis of femoral neuropathy caused by enlarged polycystic kidney was made. Cyst infection was managed with antibiotics. Renal function was maintained by frequent regular hemodialysis. While avoiding activities that may increase abdominal pressure, rehabilitation exercises were provided. Motor strength in hip flexion and knee extension improved, and was confirmed via electrodiagnostic studies.
\end{abstract}

Keywords Femoral neuropathy, Autosomal dominant polycystic kidney, Polycystic kidney diseases

\section{INTRODUCTION}

Femoral neuropathy is a rare, but unpleasant condition, characterized by quadriceps muscle weakness resulting in difficulty in knee extension and hip flexion, significantly affecting the functional outcome. Often, it is accompanied by groin pain and numbness of the antero- medial thigh. It is reported to be caused by iliacus hematoma from complications of pelvic and hip surgery [1], retroperitoneal bleeding induced by anticoagulant agents or clotting disorders [2], inguinal repair [3], or after renal transplantation [4]. Other possible etiologies include pelvic tumors [5], and pelvic radiation [6]. Unless occurred immediately after surgery, most related symptoms devel-

Received June 19, 2017; Accepted September 5, 2017

Corresponding author: Joongmo Kang

Department of Physical Medicine and Rehabilitation, Inje University Ilsan Paik Hospital, 170 Juhwa-ro, Ilsanseo-gu, Goyang 10380, Korea. Tel: +82-31910-7440, Fax: +82-31-910-7446, E-mail: stevejkang@gmail.com

ORCID: Jeehyun Yoo (http://orcid.org/0000-0002-1555-8029); Kil-Byung Lim (http://orcid.org/0000-0001-8971-7486); Hong-Jae Lee (http://orcid. org/0000-0001-9813-4952); Jiyong Kim (http://orcid.org/0000-0003-4693-8400); Eun-Cheol You (http://orcid.org/0000-0002-6435-7813); Joongmo Kang (http://orcid.org/0000-0003-3133-9202).

(c) This is an open-access article distributed under the terms of the Creative Commons Attribution Non-Commercial License (http://creativecommons.org/ licenses/by-nc/4.0) which permits unrestricted noncommercial use, distribution, and reproduction in any medium, provided the original work is properly cited. Copyright $\odot 2018$ by Korean Academy of Rehabilitation Medicine 
op occultly, and are frequently overlooked by clinicians [7].

This case report describes a patient with femoral neuropathy caused by autosomal dominant polycystic kidney disease (ADPKD). We conducted a comprehensive review of current literature available on this subject. It is the first case report of its kind.

\section{CASE REPORT}

A 55-year-old woman was diagnosed with ADPKD in 2013. Six months after the diagnosis, she started dialysis, and was undergoing hemodialysis three times weekly ever since. She had a history of recurrent cyst infection requiring hospitalization four times: May 2014, January 2015, February 2016, and July 2016. Because of the weakness, she sustained a fall in July, which resulted in left knee contusion. Knee MRI showed intact knee ligaments. She was referred to the Department of Neurology of Ilsan Paik Hospital in August 2016 for possible myopathy presenting with slowly progressing proximal lower extremity weakness. Such weakness was reported to have started 6 months ago with fluctuation, from February 2016. Physical examination revealed severe abdominal distention, and mild quadriceps muscle atrophy. Manual muscle test (MMT) right/left showed hip flexion $2 / 2+$, knee extension $2 / 2+$, ankle dorsiflexion $5 / 5$, and ankle plantarflexion $5 / 5$. Both knee deep tendon reflexes were decreased.

Nerve conduction study (NCS) was performed on the second day of admission. Femoral nerve motor conduction study showed decreased amplitude, $0.6 \mathrm{mV}$ on the right side and $1.4 \mathrm{mV}$ on the left side (Table 1). Sensory NCS for saphenous nerve revealed no response on the right side, compared with amplitude of $7.4 \mu \mathrm{V}$ on the left side (Table 2). Needle electromyography (EMG) indicated positive sharp waves and fibrillation potentials at rest in both vastus lateralis and vastus medialis muscles. Motor unit action potentials (MUAP) of these muscles displayed polyphasic morphology. Partial interference patterns were observed at maximal volition (Table 3).

Computed tomography (CT) scan was performed to identify the kidney size. It showed massively enlarged kidneys occupying the whole abdominal cavity: right kidney measuring $28.2 \mathrm{~cm}$ and left kidney $23.9 \mathrm{~cm}$ in long axis diameter due to polycystic change (Fig. 1). Pelvic magnetic resonance imaging (MRI) revealed femoral

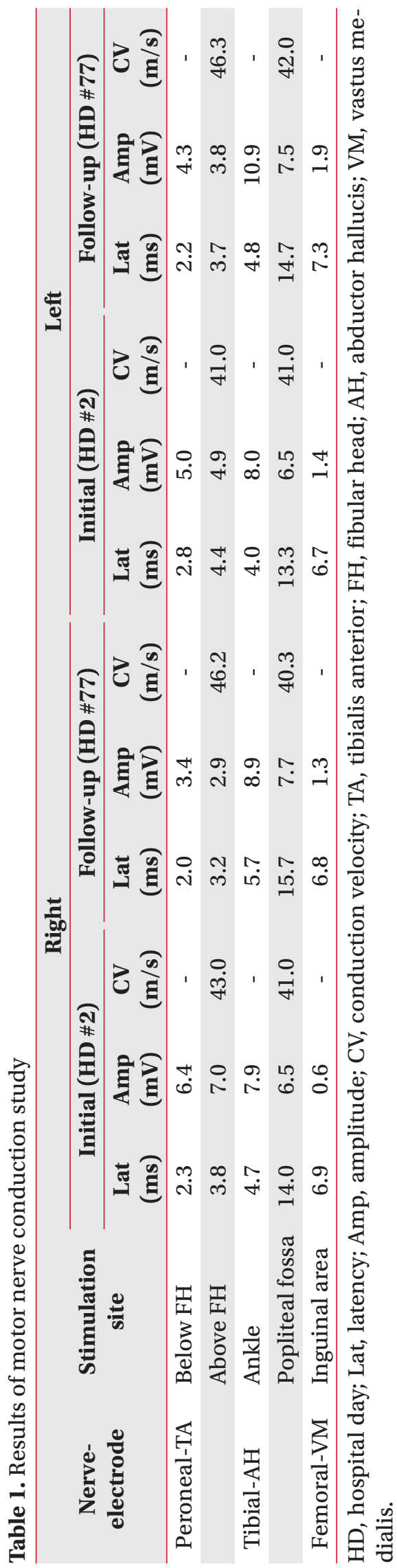

www.e-arm.org 

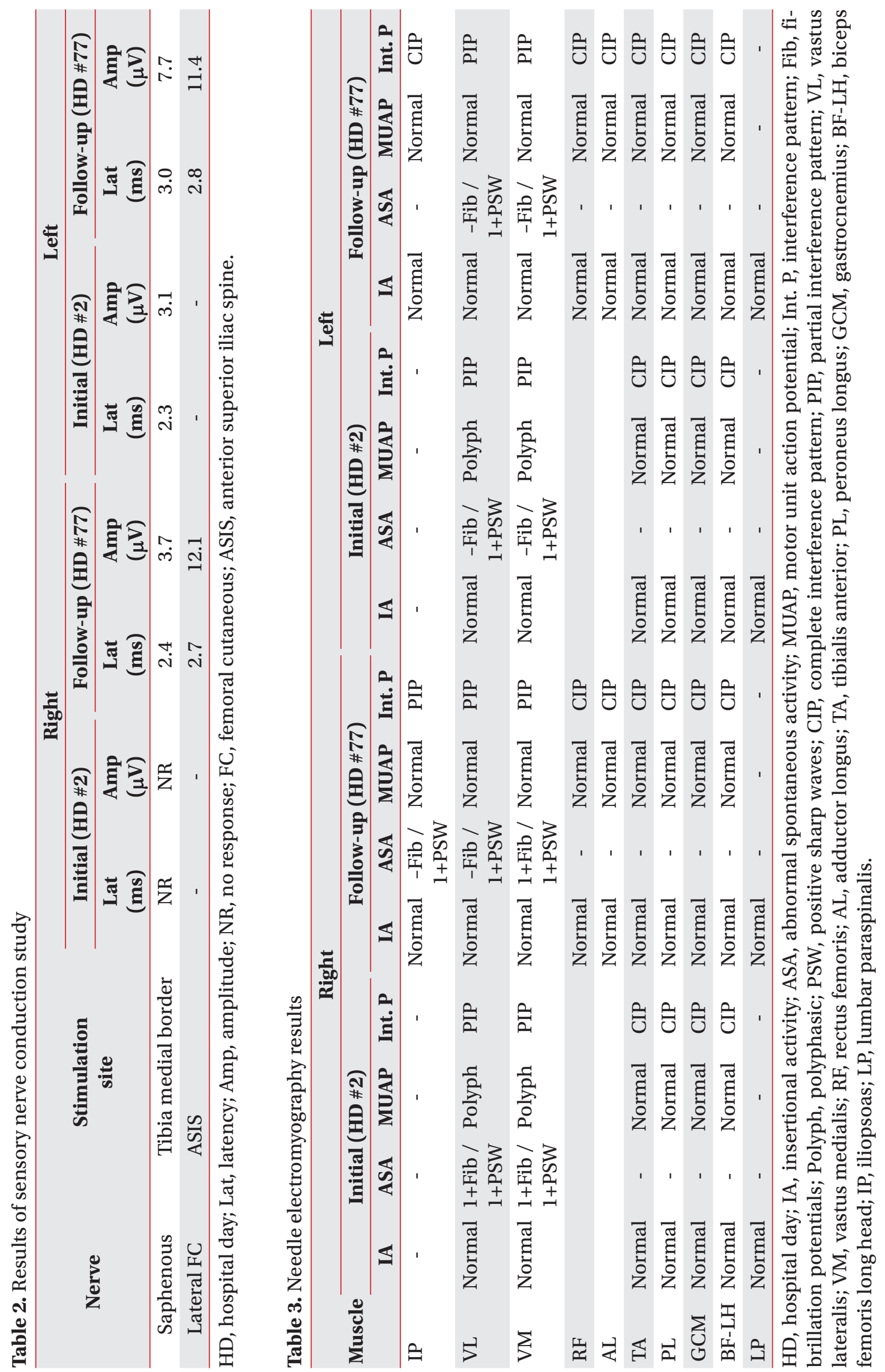

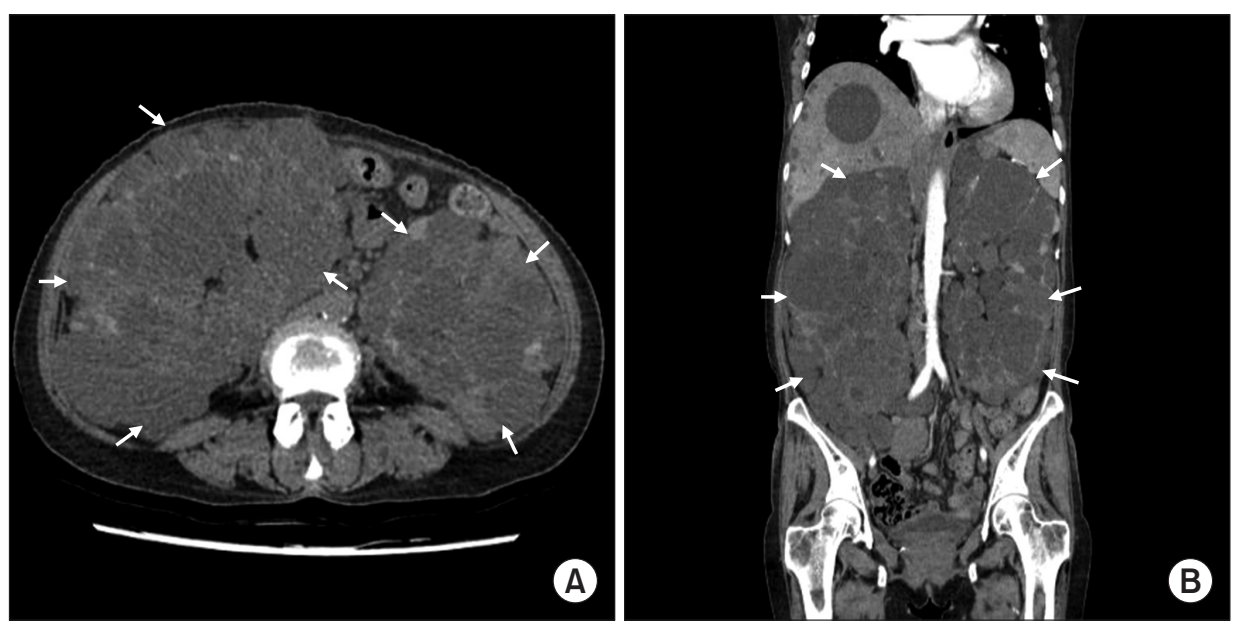

Fig. 1. Abdominal/pelvic computed tomography illustrates enormous polycystic kidney covering the entire abdominal cavity in transverse view (A) and coronal view (B).

nerve compression at the origin on the right side (Fig. 2). Femoral nerve contour and signal showed no abnormality below hip level. Based on the clinical symptoms, and findings from NCS, EMG, and MRI, a diagnosis of compressive femoral neuropathy was made. Plexopathy was excluded because the weakness, EMG and NCS findings were inconsistent. The obturator nerve was not involved, probably due to its relatively antero-medial location to the femoral nerve.

Surgery for the removal of polycystic kidney was indicated, but the patient desired to schedule the surgery later, due to surgical challenges. The patient was transferred to the Department of Physical Medicine and Rehabilitation for conservative care.

During rehabilitation, she suffered another cyst infection in October 2016. Motor weakness temporarily progressed during the episode, but after antibiotics treatment, motor strength improved. The patient underwent hemodialysis three times per week to maintain renal function. Daily education to avoid activities increasing abdominal pressure was provided. The patient was asked to avoid excessive hip flexion when sitting, and also avoid performing Valsalva maneuvers during regular activities. Straight leg raise, knee extension, and gait training exercises were added, with minimal increase in abdominal pressure. Finally, after 2 months, MMT showed improvement in right/left hip flexion 3-/3, and knee extension $3 / 3$. After all, the patient was able to ambulate with minimal assistance, and even with supervision when walking short distance.

Seventy-four days after the initial analysis, NCS and EMG were performed again. The amplitude of femo-

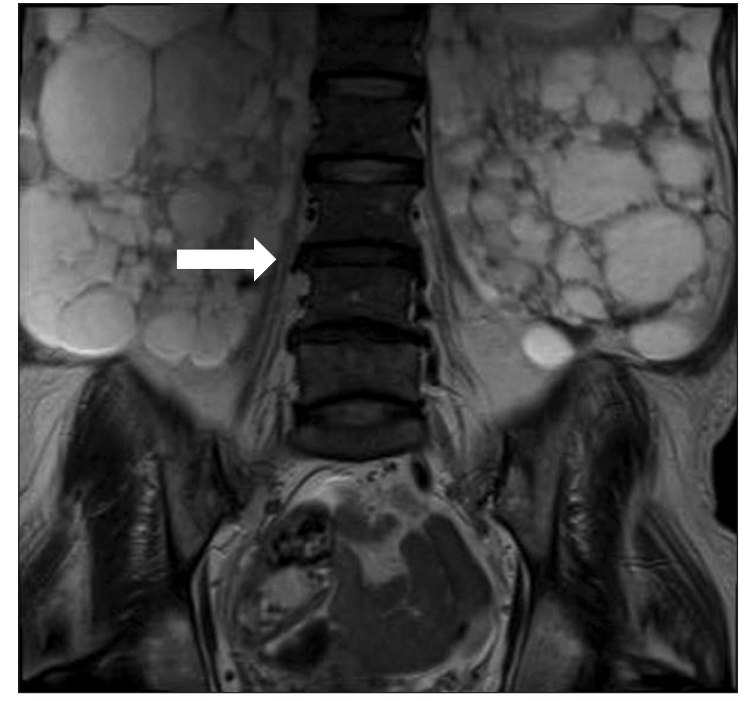

Fig. 2. Axial T2-weighted image shows femoral nerve compression by polycystic kidneys (white arrow) in coronal view. The posterior division of lumbar plexus displaying the femoral nerve origin shows irregular contour, compressed by renal cysts.

ral nerve compound muscle action potential increased from initial $0.6 \mathrm{mV}$ to $1.3 \mathrm{mV}$ on the right, and from 1.4 $\mathrm{mV}$ to $1.9 \mathrm{mV}$ on the left side (Table 1 ). While there was no response in right saphenous NCS initially, it showed improved amplitude of $3.7 \mu \mathrm{V}$. Further, the left saphenous nerve amplitude increased from $3.1 \mu \mathrm{V}$ to $7.7 \mu \mathrm{V}$ (Table 2). On the EMG study, polyphasic MUAP turned into a normal pattern, while both vastus medialis and vastus lateralis muscles showed persistent positive sharp waves (Table 3). The vastus lateralis and vastus medialis muscles, which showed reduced (partial) interference 
patterns in the initial EMG study demonstrated increased interference at follow-up, but not adequate to distinguish as full (complete) interference patterns. This result suggested insufficient motor unit recruitment and partial recovery in knee extension motor strength, from $2 / 2+$ to $3 / 3$ in MMT right/left. The functional level improved from wheelchair dependence to independent gait with minimal assistance. Unlike the initial study, fibrillation potentials were detected only in the right vastus medialis. The adductor longus muscle did not show abnormal spontaneous activities.

\section{DISCUSSION}

Polycystic kidney disease is characterized by the presence of fluid-filled renal cysts with progressive cyst formation and extensive renal enlargement that frequently results in end-stage renal disease. A study reported a patient with polycystic kidney disease who underwent bilateral nephrectomy carrying a total kidney weight of $22 \mathrm{~kg}$, which was approximately $20 \%$ of the total weight [8]. The frequently accompanying hepatic cysts in ADPKD may induce the compression of inferior vena cava, hepatic veins, or bile duct by the mass effect. The enlarged kidneys in ADPKD are known to cause intestinal obstruction by compression [9]. However, enlarged kidneys causing femoral nerve compression have never been reported. As indicated earlier, the previously reported causes of compressive femoral neuropathy include mostly iliacus hematomas after pelvic or abdominal surgery [1], or after anticoagulant use [2].

The femoral nerve is the largest branch of the lumbar plexus, which arises from the posterior division of the ventral rami of the L2-L4 spinal nerves. Initially, it passes through the psoas muscle and then between psoas and iliacus shallow groove. The nerve exits the pelvis through the femoral canal under the inguinal ligament into the femoral triangle. At a short distance below the inguinal ligament, the femoral nerve divides into muscular and cutaneous branches which are distributed to the quadriceps, pectineus, and sartorius muscles. Due to its anatomic location, femoral neuropathy is known to occur in about $2 \%$ of renal transplant patients from perioperative nerve compression and ischemia [5].

Similar to femoral neuropathy associated with postrenal transplants, direct compression on the nerve is the likely cause of femoral neuropathy in polycystic kidney disease. Other factors such as the temporary mass effect on blood vessels supplying femoral nerve may play a role in the pathogenesis. In this patient, motor weakness of the lower extremities developed in February 2016, when she was admitted for infected kidney cysts. Since then, exacerbation of motor weakness recurred intermittently, which again improved partially after antibiotic treatment. On each cyst infection episode, the size of renal cyst increased due to inflammation resulting in compression femoral nerve, which explains the reason for motor weakness aggravation after infection of kidney cysts.

In this patient, MRI failed to reveal obvious femoral nerve compression on the left side due to the following reasons. First, MRI was performed when the motor weakness was not at its peak, and when concomitant cyst infection was present. Second, motor weakness of the left lower extremities was not as severe as the right side, and therefore, relatively less distress due to left femoral nerve may not have resulted in obvious compression on the MR image.

In polycystic kidney disease, the total kidney volume indicates the extent of disease progression. Hypertension is related to progressive kidney enlargement and is an independent risk factor for progression to end-stage renal disease [10]. Therefore, controlling hypertension attenuates kidney enlargement. In this patient, by maintaining renal function with regular hemodialysis, a stable blood pressure was maintained, which may have delayed renal enlargement.

The effect of rehabilitation strengthening exercise on femoral neuropathy is not well understood. Nevertheless, Sharma et al. [5] reported that all 184 patients with acute femoral neuropathy following renal transplant surgery recovered complete motor function within 4-9 months after physical therapy. Furthermore, significant improvement in motor deficit was reported within 4-8 weeks from symptom onset [5]. Similar improvement was demonstrated in this patient. Educating the patient to avoid posture and activities resulting in increased abdominal pressure may have limited further femoral nerve compression.

In conclusion, this study is the first case report demonstrating femoral nerve compression caused by ADPKD. EMG and NCS should be considered for polycystic kidney disease patients with weak hip flexion and knee ex- 
tension. CT and MRI can assist in further identifying the etiology. Symptoms may progress due to infected renal cysts. Maintenance of renal function and management of cyst infection is crucial to accelerate recovery. Rehabilitation exercises and avoidance of aggravating posture and activities may prevent exacerbation of nerve compression.

\section{CONFLICT OF INTEREST}

No potential conflict of interest relevant to this article was reported.

\section{REFERENCES}

1. Kumar S, Pflueger G. Delayed femoral nerve palsy associated with iliopsoas hematoma after primary total hip arthroplasty. Case Rep Orthop 2016;2016:6963542.

2. Ho KJ, Gawley SD, Young MR. Psoas haematoma and femoral neuropathy associated with enoxaparin therapy. Int J Clin Pract 2003;57:553-4.

3. Lange B, Langer C, Markus PM, Becker H. Paralysis of the femoral nerve following totally extraperitoneal laparascopic inguinal hernia repair. Surg Endosc 2003; 17:1157.
4. Sharma KR, Cross J, Santiago F, Ayyar DR, Burke G. Incidence of acute femoral neuropathy following renal transplantation. Arch Neurol 2002;59:541-5.

5. Hsu YC, Shih YY, Gao HW, Huang GS. Intramuscular schwannoma arising from the psoas muscle presenting with femoral nerve neuropathy. South Med J 2010; 103:477-9.

6. Mendes DG, Nawalkar RR, Eldar S. Post-irradiation femoral neuropathy: a case report. J Bone Joint Surg Am 1991;73:137-40.

7. Huang WS, Lin PY, Yeh CH, Chin CC, Hsieh CC, Wang JY. Iatrogenic femoral neuropathy following pelvic surgery: a rare and often overlooked complication--four case reports and literature review. Chang Gung Med J 2007;30:374-9.

8. Ekser B, Rigotti P. Images in clinical medicine: autosomal dominant polycystic kidney disease. N Engl J Med 2010;363:71.

9. Carels RA, van Bommel EF. Ruptured giant liver cyst: a rare cause of acute abdomen in a haemodialysis patient with autosomal dominant polycystic kidney disease. Neth J Med 2002;60:363-5.

10. Chapman AB, Stepniakowski K, Rahbari-Oskoui F. Hypertension in autosomal dominant polycystic kidney disease. Adv Chronic Kidney Dis 2010;17:153-63. 